\title{
Catalytic performance of surface modified-niobic acid
}

\author{
Adid Adep Dwiatmoko* \\ Clean Energy and Chemical Engineering, University of Science and Technology, Daejeon, South Korea \\ ( ${ }^{\#}$ Current affiliation: Research Center for Chemistry, Indonesian Institute of Sciences, 15314, Tangerang Selatan, Indonesia)
}

\begin{abstract}
Surface modification of niobic acid with phosphoric acid and its catalytic performance in the hydrolysis reaction has been studied. A series of catalysts, surface-modified with different concentration of phosphoric acid, have been prepared and characterized. It was found that the catalyst selectivity to glucose increased with the increase of acid concentration used for the surface modification. The extent of selectivity enhancement of the modified catalysts showed a good relationship with the atomic ratio of phosphorous and niobium on the surface.
\end{abstract}

\section{Introduction}

Since the first report by Tanabe [1], niobic acid (NA) has been recognized to be an effective catalyst for several reactions involved with participation or liberation of water molecules $[2,3]$. It is an insoluble polymeric oxide with different amounts of hydrated water depending on a preparation method [1]. The surface of niobic acid generally exhibits a high acid strength, even in the presence of water, as long as it remains in an amorphous form [4]. Moreover, calcination or acid treatment makes its acid properties to be tuned in a certain manner [5]. Especially, the treatment with mineral acids has been reported to be effective in suppressing the crystallization of niobic acid and increasing its surface acidity as well [6]. More recently, we have synthesized phosphoric acidtreated niobic acid catalyst exhibited high activities for hydrolysis of disaccharides to glucose in water, where the conversion and glucose selectivity were $52 \%$ and $82 \%$, respectively [7]. However, discussion on the relationship between its catalytic activity and acid property was not fully made in the above report.

Herein we report the investigation into catalytic behaviors of niobic acid, modified with different concentrations of phosphoric acid on the hydrolysis of cellobiose. Thus, expected products formed from cellobiose would be glucose, fructose, HMF and organic acids (levulinic acid and formic acid). Aside from these products identified, some unidentified products could be produced. Since one of major concerns in this case is a higher selectivity of a certain product, this work was focused on finding an active and selective phosphoric acid-treated niobic acid catalyst for cellobiose conversion. From the result that glucose, instead of HMF or organic acids, was favorably produced using the catalyst; an indepth explanation was formulated for unveiling the effect of phosphoric acid treatment on enhancing the selectivity of glucose.

\footnotetext{
* Corresponding author: adid.adep.dwiatmoko@lipi.go.id
}

\section{Experimental}

\subsection{Catalyst preparation}

Niobic acid was modified with different concentration of phosphoric acid (x), denoted as $\mathrm{P}-\mathrm{NA}(\mathrm{x})$ in which $\mathrm{x}$ is the acid concentration $(0.5,1$, and 2$)$ in a unit of $\mathrm{mol} / \mathrm{L}$, following the method described elsewhere [7, 8]. Briefly, niobic acid ( $1 \mathrm{~g})$, utilized as an as-received form, was immersed in $20 \mathrm{~mL}$ of phosphoric acid solution with a specified concentration and stirred at room temperature for $72 \mathrm{~h}$. The suspension was then filtered and thoroughly washed with large amounts of distilled water until a neutral $\mathrm{pH}$ was achieved. The resulting material was subsequently dried in the oven at $100{ }^{\circ} \mathrm{C}$ overnight.

\subsection{Catalyst characterization}

The phase structure of prepared catalysts was observed by a powder X-ray diffraction (XRD) analysis using a Rigaku X-ray diffractometer with $\mathrm{Cu} \mathrm{K} \alpha(\lambda=0.15406 \mathrm{~nm})$ radiation source operated at $40 \mathrm{kV}$ and $30 \mathrm{~mA}$, where $2 \theta$ value was in the range of $5-90^{\circ}$. The surface $\mathrm{P}$ and $\mathrm{Nb}$ species were characterized by X-ray photoelectron spectroscopy (XPS) using a PHI 5000 VersaProbe (Ulvac-PHI) analytical instrument with a monochromator

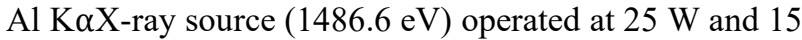
$\mathrm{kV}$ with the spot size of $100 \mu \mathrm{m}$ by $100 \mu \mathrm{m}$. The binding energies of the XPS data were referenced to the $\mathrm{C} 1 \mathrm{~s}$ peak at $284.6 \mathrm{eV}$ with an accuracy of $0.1 \mathrm{eV}$ and charge neutralization was employed.

\subsection{Catalytic Reaction}

In order to evaluate the catalytic performances of prepared catalysts, a mixture containing cellobiose $(0.2 \mathrm{~g})$, distilled water $(20 \mathrm{~mL})$, catalyst $(0.1 \mathrm{~g})$ was prepared and heated 
with stirring in a stainless-steel autoclave under a $\mathrm{N}_{2}$ atmosphere at $140{ }^{\circ} \mathrm{C}$. After the reaction for $4 \mathrm{~h}$, the mixture was cooled to room temperature and then filtered for analysis of products. The final concentrations of identified products were quantitatively determined by a high-performance liquid chromatography using a BioRad HPX-87H column $(7.8 \times 300 \mathrm{~mm})$ at $35^{\circ} \mathrm{C}$ equipped with a refractive index detector (Young Lin YL 9170 RID), where the mobile phase was $0.005 \mathrm{M}$ sulfuric acid and the flow rate was $0.5 \mathrm{~mL} / \mathrm{min}$.

\section{Results and Discussion}

The activity of the prepared catalysts was evaluated in cellobiose hydrolysis reaction. Cellobiose, a model substrate of cellulose, is converted to glucose through hydrolysis reaction, followed by dehydration of water to form HMF in acid-catalyzed reaction. Furthermore, in the presence of acid species, HMF can be converted to organic acids, such as levulinic acid and formic acid. Polymer products and insoluble humins can be generated from these products by recombination with sugars or oligosaccharides via aldol condensation [9].

The effect of phosphoric acid concentration employed for surface modification of niobic acid was examined at a concentration ranging from 0.5 to $2 \mathrm{~mol} / \mathrm{L}$. As shown in Figure 1, the activity of the catalysts was dependent on the concentration of phosphoric acid utilized. When the concentration of phosphoric acid was $0.5 \mathrm{~mol} / \mathrm{L}$, the cellobiose conversion was $20 \%$. Furthermore, at a higher concentration of $1 \mathrm{~mol} / \mathrm{L}$, the cellobiose conversion increase up to $39.6 \%$. Further increase of the concentration of phosphoric acid up to $2 \mathrm{~mol} / \mathrm{L}$ did not lead to the increase of the catalyst activity. Interestingly, the selectivity of glucose showed a similar tendency with the trend of catalyst activity. In the acid concentration of $0.5 \mathrm{~mol} / \mathrm{L}$, the selectivity of glucose was $36.1 \%$, and it significantly improved up to $82.7 \%$ by increasing acid concentration to $1 \mathrm{~mol} / \mathrm{L}$. This selectivity does not significantly change by further increase of phosphoric acid concentration. In addition, the formation of HMF were not observed, which was somewhat inhibited by the surface modification of phosphoric acid on the catalysts.

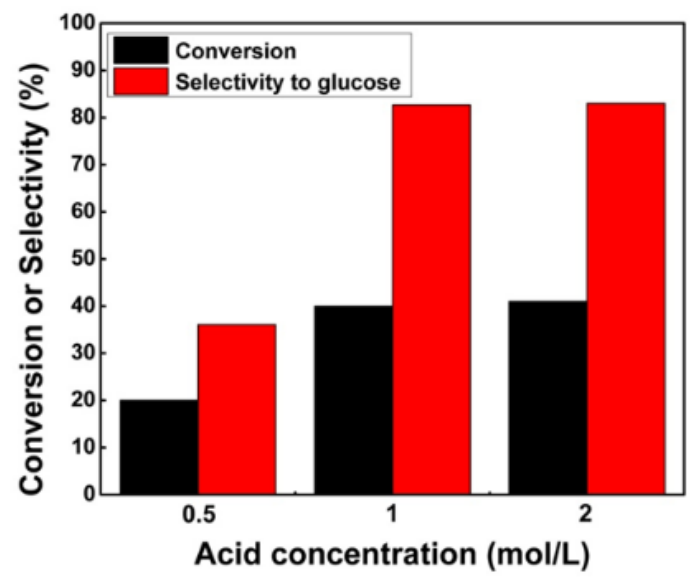

Fig. 1. Effect of phosphoric acid concentration on the catalytic activity of surface-modified niobic acid catalysts in the hydrolysis of cellobiose.
$\mathrm{XRD}$ analysis was performed to examine the effect of the surface modification on the structural change of the catalysts. Figure 2 shows the XRD patterns of the prepared catalysts, P-NA(x). Niobic acid (NA), which has an amorphous structure, shows only broad diffraction peaks at $2 \theta=14.5^{\circ}, 28.7^{\circ}, 49.1^{\circ}$ and $57.7^{\circ}$. When phosphoric acid was applied for modification with concentration of $1 \mathrm{~mol} / \mathrm{L}$ or higher, these peaks was either weaken or relieved, and then, clear diffractions at $2 \theta=$ $11.8^{\circ}, 14.5^{\circ}, 19.6^{\circ}, 22.9^{\circ}, 27.8^{\circ}, 27.8^{\circ}, 39.7^{\circ}, 44.4^{\circ}$, $57.2^{\circ}, 60.9^{\circ}$, and $64.7^{\circ}$ started to appear, indicating the formation of a crystalline phase of niobium phosphate structure occurred [6]. This result suggested that niobium phosphate was formed on the surface of niobic acid during introduction with phosphoric acid at concentration of equal to or higher than $1 \mathrm{~mol} / \mathrm{L}$.

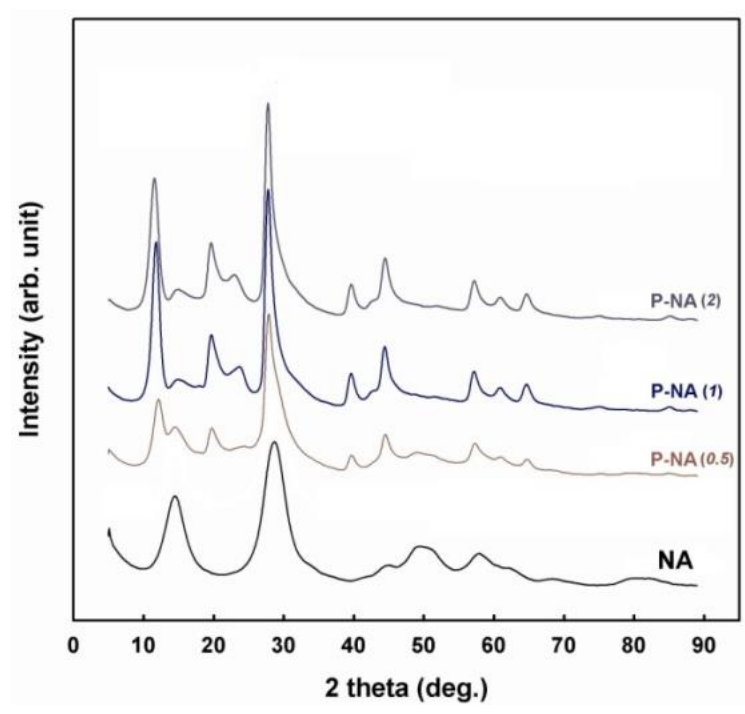

Fig. 2. XRD patterns of the prepared catalysts.

Figure 3 shows the XPS patterns of phosphorus species on the catalysts surface. No binding energy peak of P2p was observed on the original niobic acid (NA) due to the absence of the surface modification with phosphoric acid. XPS spectras of P2p for the surface-modified catalysts show that with an increase in concentration of phosphoric acid, the XPS intensity of the P2p peak on the modified catalysts increases. The P $2 p$ XPS spectra of the catalysts showed the presence of phosphate ions of $\mathrm{H}_{2} \mathrm{PO}_{4}^{-}$, which corresponds to a signal centered at around $133.0 \pm 1.0 \mathrm{eV}$ with a full width at half maximum of $2.1 \mathrm{eV}$ $[10,11]$, consistent with the result from XRD analysis. A small peak at the binding energy around $133.0 \mathrm{eV}$ can be detected for the P-NA(0.5) catalyst. This binding energy was gradually shifted to a higher binding energy with an increase in concentration of phosphoric acid employed. A high-intensity peak was recorded at the binding energy of $133.5 \mathrm{eV}$ for the P-NA(2) catalyst. 


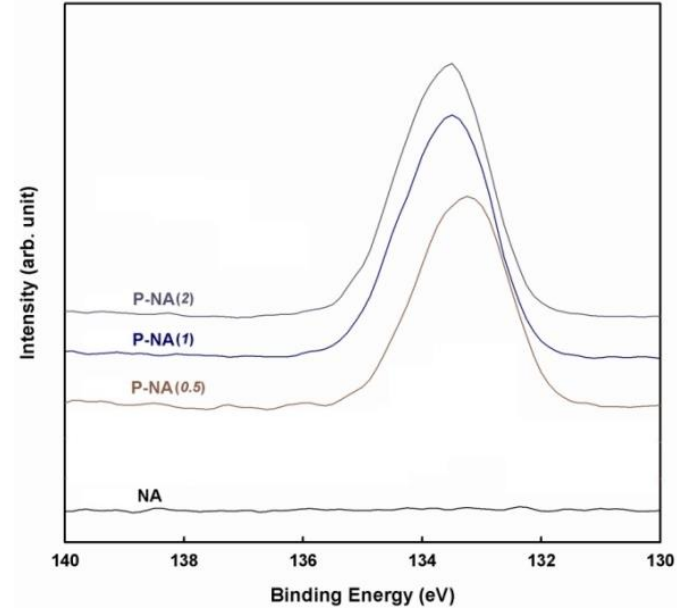

Fig. 3. XPS spectra characterizing P2p on the surface of the catalysts.

Furthermore, as shown in Table 1, the atomic concentration of niobium, oxygen and phosphorous on the catalysts surface as a function of phosphoric acid employed during modification was measured by XPS. The atomic ratio of phosphorous and niobium $(\mathrm{P} / \mathrm{Nb})$ was then calculated. In the absence of the surface modification, the phosphorous was not detected, so that the ratio of $\mathrm{P} / \mathrm{Nb}$ was not applicable. As the concentration of phosphoric acid increased from $0.5 \mathrm{~mol} \cdot \mathrm{L}-1$ to $1 \mathrm{~mol} \cdot \mathrm{L}-$ 1 , the ratio of $\mathrm{P} / \mathrm{Nb}$ increased from 0.89 to 1.11 . Further increase of phosphoric acid concentration up to $2 \mathrm{~mol} \cdot \mathrm{L}-$ 1 , did not lead to the increase of the $\mathrm{P} / \mathrm{Nb}$ ratio. Based on this observation, it is suggested that the interaction of phosphate group on the catalyst surface was saturated at 1 mol-L-1 of phosphoric acid employed during the surface modification. Increasing the concentration of phosphoric acid, would not affect the number of phosphate groups attached on the catalyst surface.

Table 1. Surface concentration of the catalysts.

\begin{tabular}{|c|c|c|c|c|c|}
\hline \multirow{2}{*}{ Catalyst } & \multicolumn{3}{|c|}{ Atomic concentration (\%) } & \multirow{2}{*}{$\mathbf{P} / \mathbf{N b}$} & \multirow{2}{*}{$\mathbf{O} / \mathbf{N b}$} \\
\hline & $\mathbf{N b}$ & $\mathbf{O}$ & $\mathbf{P}$ & & \\
\hline$\overline{\mathrm{NA}}$ & 14.76 & 39.99 & - & - & 2.71 \\
\hline $\mathrm{P}-\mathrm{NA}(0.5)$ & 10.38 & 62.37 & 9.21 & 0.89 & 6.01 \\
\hline P-NA(1) & 9.15 & 60.50 & 10.16 & 1.11 & 6.61 \\
\hline $\mathrm{P}-\mathrm{NA}(2)$ & 10.09 & 65.93 & 10.96 & 1.09 & 6.53 \\
\hline
\end{tabular}

The above mentioned results clearly demonstrated that the surface modification of niobic acid catalyst with mineral acids enhanced the catalyst selectivity to glucose. It was found that the selectivity of phosphoric acidmodified niobic acid was significantly higher than that of obtained with sulfuric acid-modified catalyst. HMF and organic acids were still obtained during the reactions. It is well-known that the acidic property of the catalyst could be modified by introducing mineral acids onto its surface. Thus, the possible explanation of the different results obtained using $\mathrm{H}_{3} \mathrm{PO}_{4}-\mathrm{Nb}_{2} \mathrm{O}_{5}$ and $\mathrm{H}_{2} \mathrm{SO}_{4}-\mathrm{Nb}_{2} \mathrm{O}_{5}$ could be related to its acid property. It was suggested that the formation of further products, such as HMF and organic acids could be related to the difference in the surface acidity of the catalysts. Based on that, we determined the acid strength of the modified catalysts by Hammett indicator. After being modified with $1 \mathrm{~mol} \cdot \mathrm{L}^{-1}$ phosphoric acid, its $\mathrm{H}_{\mathrm{o}}$ value was $-5.6<\mathrm{H}_{\mathrm{o}} \leq-3.3$. Interestingly, the $\mathrm{H}_{\mathrm{o}}$ values of the modified catalyst with $1 \mathrm{~mol} \cdot \mathrm{L}^{-1} \mathrm{H}_{3} \mathrm{SO}_{4}$ was found to be $-8.2<\mathrm{Ho} \leq-5.6$. This shows that the $\mathrm{H}_{2} \mathrm{SO}_{4}-\mathrm{Nb}_{2} \mathrm{O}_{5}$ has a stronger acid sites compared to the $\mathrm{H}_{3} \mathrm{PO}_{4}-\mathrm{Nb}_{2} \mathrm{O}_{5}$, which lead to the formation of other products from cellobiose hydrolysis, aside from glucose. This was also supported by the fact that sulfuric acid $\left(\mathrm{pK}_{\mathrm{a}}\right.$ $=-3)$ is a stronger mineral acid than phosphoric acid $\left(\mathrm{pK}_{\mathrm{a}}\right.$ $=2.12$ ).

Taking into account that the XRD analysis shows that the niobium phosphate was formed on the catalyst surface, the surface reaction which occurs between phosphoric acid and niobic acid could be described as depicted in Figure 4. This attached ions on the niobic acid surface acts as additional active and selective sites to convert cellobiose to glucose.

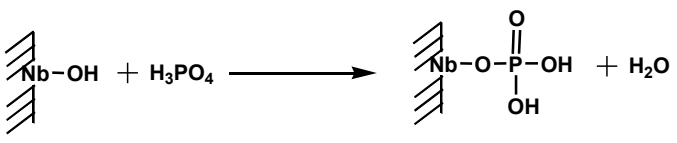

Fig. 4. Interaction between phosphoric acid and catalyst surface.

Another issue in the heterogeneous catalytic system is in regards to the catalyst stability. Since the stability of the catalyst is also an important issue in heterogeneous catalysis, we did carry out the recyclability test of the modified catalyst in our catalytic reaction. Figure 5 shows the experimental results of the recyclability of the $\mathrm{H}_{3} \mathrm{PO}_{4}-$ $\mathrm{Nb}_{2} \mathrm{O}_{5}(2)$ catalyst in the hydrolysis of cellobiose five runs. After each run, the catalyst was separated using centrifuge, and subsequently used without any treatment before being applied for the next catalytic reaction. As shown in the figure, the average cellobiose conversion was about $31.3 \%$ with the average glucose yield of $25.3 \%$. Even if the active species might leach out of the catalyst surface, there was no significant change in the activity of the catalyst after being used for five runs. Obviously, the catalyst is a sufficiently stable and highly selective for the conversion of cellobiose under our reaction conditions.

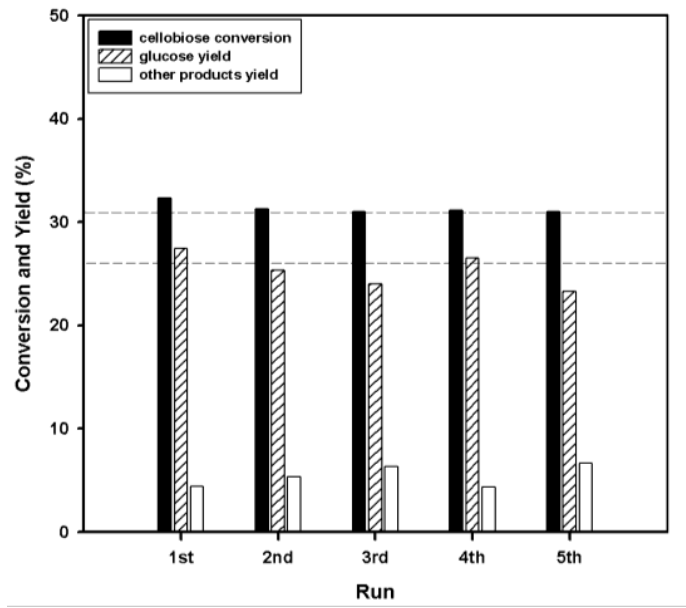

Fig. 5. Stability test of the prepared catalyst. 


\section{Conclusions}

We have demonstrated in this work, that the surface modification of the niobic acid catalyst $\left(\mathrm{Nb}_{2} \mathrm{O}_{5} \cdot \mathrm{xH}_{2} \mathrm{O}\right)$ with phosphoric acid has improved the selectivity in the formation of glucose from cellobiose hydrolysis. Selectivity of glucose increased up to $82.7 \%$ with the increase of phosphoric acid concentration used for surface modification up to $1 \mathrm{~mol} \cdot \mathrm{L}^{-1}$. Further increase of phosphoric acid concentration did not lead to substantial changes in both the glucose yield and selectivity. The extent of selectivity enhancement of the modified catalysts showed a good relationship with the atomic ratio of phosphorous and niobium on the surface measured by XPS. It is believed that the principal contributor to the selectivity enhancement is the presence of dihydrogenphosphate $\left(\mathrm{H}_{2} \mathrm{PO}_{4}^{-}\right)$as an active species on the catalysts surface formed from the reaction which occurs between phosphoric acid and niobic acid, confirmed by XPS. This study shows the surface modification of niobic acid catalysts promotes the formation of valuable products from cellobiose and, further, lignocellulosic materials.

This work was done by the financial support of Korea Institute of Science and Technology at Korea Institute of Science and Technology when the author studied. Guidance and supervision of Prof. Young-Woong Suh and Prof. Dong Jin Suh are greatly acknowledged. The results have been presented by Prof. Dong Jin Suh in Europacat X.

\section{References}

1. Tanabe, K., Niobic acid as an unusual acidic solid material. Materials chemistry and physics, 1987. 17(1-2): p. 217-225.

2. Doiseau, A., F. Rataboul, and N. Essayem. Xylose dehydration in furfural using a continuous fix-bed reactor: Insights on phosphate addition to niobic acid performances.

3. Reche, M.T., et al., Niobic acid nanoparticle catalysts for the aqueous phase transformation of glucose and fructose to 5-hydroxymethylfurfural. Catalysis Science \& Technology, 2016. 6(19): p. 7334-7341.

4. Chen, Z.-h., T. Iizuka, and K. Tanabe, Niobic acid as an efficient catalyst for vapor phase esterification of ethyl alcohol with acetic acid. Chemistry Letters, 1984. 13(7): p. 1085-1088.

5. Shishido, T., et al., Brønsted acid generation over alumina-supported niobia by calcination at $1173 \mathrm{~K}$. Catalysis letters, 2009. 129(3-4): p. 383-386.

6. Okazaki, S., et al., The effect of phosphoric acid treatment on the catalytic property of niobic acid. Bulletin of the Chemical Society of Japan, 1987. 60(1): p. 37-41.

7. Dwiatmoko, A.A., Rinaldi, N., Preparasi dan Karakterisasi Katalis Asam Padat Berbasis Niobia untuk Produksi Senyawa Glukosa. Research Journal of Industrial Pollution Prevention Technology, 2017. Submitted.
8. Okazaki, S. and A. Kurosaki, Acidic properties and catalytic activities of niobic acid treated with phosphoric acid. Catalysis Today, 1990. 8(1): p. 113122.

9. Shimizu, K.-i., et al., Effects of Brønsted and Lewis acidities on activity and selectivity of heteropolyacidbased catalysts for hydrolysis of cellobiose and cellulose. Green Chemistry, 2009. 11(10): p. $1627-$ 1632.

10. Yang, F., et al., Conversion of biomass into 5hydroxymethylfurfural using solid acid catalyst. Bioresource technology, 2011. 102(3): p. 3424-3429.

11. de Pietre, M.K., et al., H3PO4-and H2SO4-treated niobic acid as heterogeneous catalyst for methyl ester production. Reaction Kinetics, Mechanisms and Catalysis, 2010. 99(2): p. 269-280. 